\title{
X-ray emitting structures in the Vela SNR: Ejecta anisotropies and progenitor stellar wind residuals
}

\author{
V. Sapienza ${ }^{1,2}$, M. Miceli ${ }^{1,2}$, G. Peres ${ }^{1,2}$, F. Bocchino ${ }^{2}$, S. Orlando ${ }^{2}$, E. Greco ${ }^{1,2}$, J. A. Combi ${ }^{3,4}$, F. García ${ }^{5}$, and M. \\ Sasaki $^{6}$
}

\begin{abstract}
1 Dipartimento di Fisica e Chimica E. Segrè, Università degli Studi di Palermo, Piazza del Parlamento 1, 90134, Palermo, Italy
2 INAF-Osservatorio Astronomico di Palermo, Piazza del Parlamento 1, 90134, Palermo, Italy

3 Instituto Argentino de Radioastronomía (CCT-La Plata, CONICET; CICPBA; UNLP), C.C. No. 5, 1894 Villa Elisa, Argentina

${ }_{5}^{4}$ Facultad de Ciencias Astronómicas y Geofísicas, Universidad Nacional de La Plata, Paseo del Bosque s/n, 1900 La Plata, Argentina

5 Kapteyn Astronomical Institute, University of Groningen, PO BOX 800, NL-9700 AV Groningen, the Netherlands

${ }^{6}$ Dr. Karl Remeis Observatory and ECAP, Universität Erlangen-Nürnberg, Sternwartstr. 7, 96049 Bamberg, Germany
\end{abstract}

\begin{abstract}
Context. The Vela supernova remnant (SNR) shows several ejecta fragments (or shrapnel) protruding beyond the forward shock, which are most likely relics of anisotropies that developed during the supernova (SN) explosion. Recent studies have revealed high Si abundance in two shrapnel (shrapnel A and G), located in opposite directions with respect to the SNR center. This suggests the possible existence of a Si-rich jet-counterjet structure, similar to that observed in the SNR Cassiopea A.

Aims. We analyzed an XMM-Newton observation of a bright clump, behind shrapnel $\mathrm{G}$, which lies along the direction connecting shrapnel A and G. The aim is to study the physical and chemical properties of this clump to ascertain whether it is part of this putative jet-like structure.

Methods. We produced background-corrected and adaptively-smoothed count-rate images and median photon energy maps, and performed a spatially resolved spectral analysis.

Results. We identified two structures with different physical properties. The first one is remarkably elongated along the direction connecting shrapnel A and G. Its X-ray spectrum is much softer than that of the other two shrapnel, to the point of hindering the determination of the $\mathrm{Si}$ abundance; however, its physical and chemical properties are consistent with those of shrapnel A and shrapnel G. The second structure, running along the southeast-northwest direction, has a higher temperature and appears similar to a thin filament. By analyzing the ROSAT data, we have found that this filament is part of a very large and coherent structure that we identified in the western rim of the shell.

Conclusions. We obtained a thorough description of the collimated, jet-like tail of shrapnel G in Vela SNR. In addition we discovered a coherent and very extended feature roughly perpendicular to the jet-like structure that we interpret as a signature of an earlier interaction of the remnant with the stellar wind of its progenitor star. The peculiar $\mathrm{Ne} / \mathrm{O}$ ratio we found in the wind residual may be suggestive of a Wolf-Rayet progenitor for Vela SNR, though further analysis is required to address this point.
\end{abstract}

Key words. ISM: supernova remnants - ISM: individual objects: Vela SNR - X-ray: ISM

\section{Introduction}

Core collapse supernova remnants (SNRs) show complex morphologies that result from intrinsic asymmetries in the supernova (SN) explosion and from the propagation of the explosion shock-waves in very inhomogeneous environments, such as preexisting stellar winds and molecular clouds. Therefore, it is difficult to distinguish the role played by the interstellar medium (ISM) inhomogenities from that played by pristine anisotropies in the ejecta in shaping the remnant morphology. X-ray observations of SNRs are useful diagnostic tools to trace the distribution of the physical and chemical properties of the emitting ejecta and a starting point to reconstruct the details of the explosion mechanism and the structure of the ambient environment surrounding the exploded star.

Vela SNR, the relic from the explosion of a massive star that occurred $\sim 11$ kyr ago (Taylor et al. 1993), is an interesting target to study especially because of its proximity: With a distance of only $280 \mathrm{pc}$ (Dodson et al. 2003), it is the nearest SNR. This makes it possible to resolve the $\mathrm{X}$-ray emission of small-scale structures spatially and to identify the ejecta to study their properties.

Aschenbach et al. (1995) identified six X-ray emitting bowshaped ejecta fragments in regions beyond the forward shock called "shrapnel,"named from A to F (see Fig. 1). X-ray emitting ejecta have also been observed inside (in projection) the shell (Miceli et al. 2008a, LaMassa et al. 2008, Slane et al. 2018). Shrapnel A, which is one of the most distant ejecta from the explosion center, exhibits a pattern of heavy element abundances that are different from that observed in all the other ejecta. Katsuda \& Tsunemi (2006) determined the abundances of the shrapnel by finding a nearly solar abundance for $\mathrm{Ne}, \mathrm{O}, \mathrm{Mg}$ and $\mathrm{Fe}$, and overabundant $\mathrm{Si}$. The latter is expected to be produced in deeper layers of the progenitor star compared to lower $\mathrm{Z}$ elements such as $\mathrm{O}, \mathrm{Ne}$, and $\mathrm{Mg}$. Therefore, Si-rich ejecta are not expected to be so distant from the explosion center of the remnant, indicating that an inversion of ejecta layers occurred at some point during the remnant evolution. Miceli et al. (2013) show, with dedicated 2-D hydrodynamic simulations, that velocity and density contrast with respect to the surrounding ejecta 


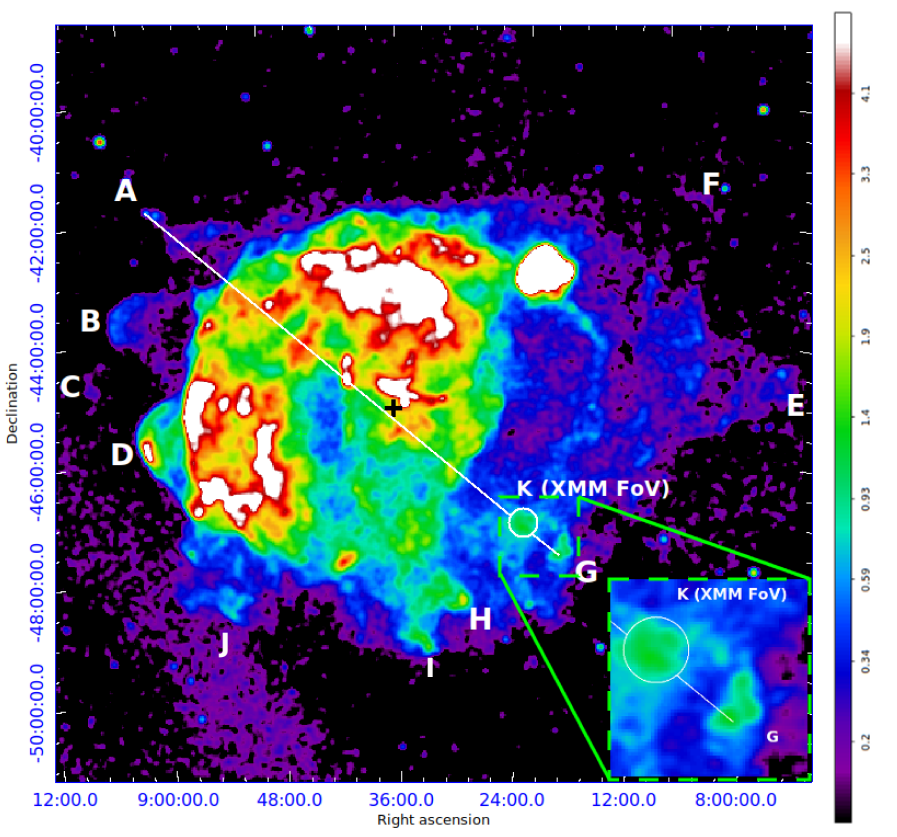

Fig. 1. ROSAT All Sky Survey (RASS) count image of Vela SNR in the $0.1-2.4 \mathrm{keV}$ energy band in squared root scale. The circle $\mathrm{K}$ is the region analyzed in this work, marking the XMM-Newton field of view. The black cross indicates the position of the Vela pulsar at the explosion time by considering an age of $11000 \mathrm{yr}$ and taking into account the pulsar proper motion estimated by Caraveo et al. (2001). We assume it as the explosion center. The inset is a close-up view of the same image.

are necessary to make the Si-rich shrapnel overtake the other shrapnel and the forward shock. More recent 3-D magnetohydrodynamic simulations confirmed the important role played by explosion asymmetries in determining a spatial inversion of ejecta layers (Orlando et al.2016, Tutone et al.2020 and Orlando et al. 2021).

García et al. (2017) analyzed an X-ray luminous knot, named shrapnel G (see Fig. 1), in the southwestern region of Vela SNR. Shrapnel $G$ is located in the anticenter with respect to shrapnel A. García et al. (2017) found that the chemical composition of shrapnel $G$ is very similar to that of shrapnel A. This suggests that shrapnel $\mathrm{A}$ and $\mathrm{G}$ are part of a jet-counterjet-like event which has throw away the inner layer of the progenitor star and has made them overcome lighter ejecta and the forward shock (somehow similar to that observed in Cassiopea A, Vink 2004). To confirm that shrapnel A and G are part of a Si-rich jet-like structure, it is necessary to ascertain the nature of the Si emission and to study the region between the two ejecta knots in detail.

The ROSAT image (see Fig. 1) of the whole Vela SNR shows that shrapnel $\mathrm{G}$ is followed by another bright clump, labeled "knot K." This knot seems to lay along the line connecting shrapnel A, shrapnel G, and the center of the SNR. This suggests that knot $\mathrm{K}$ could be physically linked to shrapnel G, which would prove the existence of a coherent $\mathrm{Si}$-rich feature on a large spatial scale.

In this paper, we present the analysis of an XMM-Newton observation of knot $\mathrm{K}$ to study its physical and chemical properties, and to ascertain whether it is part of a jet-like structure linking shrapnel A and G. We complement the analysis of the $X M M$-Newton data with ROSAT archive observations from the western part of Vela SNR's shell.

The paper is organized as follows: In Sect. 2 we present the data and their analysis; Sect. 3 and Sect. 4 show the results of
XMM-Newton and ROSAT data analysis, respectively. Finally, we discuss our results in Sect. 5.

\section{Observations and data analysis}

We analyze an XMM-Newton European Photon Imaging Camera (EPIC) observation of Vela SNR knot K. EPIC consists of a set of three X-ray sensing CCD cameras: two MOS detectors (Turner et al. 2001) and a pn detector (Strüder et al. 2001), operating in the $0.3-10 \mathrm{keV}$ band. The XMM-Newton observation was performed from October 07, 2019 to October 08, 2019 (Obs. ID 0841510101, PI: M. Miceli), with pointing coordinates $\alpha_{J 2000}=08^{h} 23^{m} 32.49^{s}$ and $\delta_{J 2000}=-47^{\circ} 11^{\prime} 55.8^{\prime \prime}$, medium filter, in full frame mode. The exposure times are $55 \mathrm{ks}, 55 \mathrm{ks}$, and $51 \mathrm{ks}$ for the MOS1, MOS2, and PN cameras, respectively. We processed the data with the Science Analysis System (SAS) software, version 18.0.0.

EPIC event files are typically contaminated by soft-protons, that is to say mildly relativistic protons that are detected by the CCD cameras. We filtered the event lists for soft-proton contamination with the espfilt task, thus obtaining a screened exposure time of approximately $33 \mathrm{ks}$ for the MOS1, $37 \mathrm{ks}$ for the MOS2, and $19 \mathrm{ks}$ for the pn camera (only $37 \%$ of the total exposure). We then filtered the event lists retaining only events with $\mathrm{FLAG}=0$ and PATTERN $\leq 4,12$ for $\mathrm{pn}$ and MOS cameras, respectively. We adopted a source detection procedure, using the edetect_chain SAS task to remove events in circular regions (with radius 18") around point-like sources.

All images were background subtracted by adopting the double subtraction procedure described in Miceli et al. (2017) to take instrumental, particle, and X-ray background contamination into account. For this purpose, we used the Filter Wheel Closed (FWC) and Blank Sky (BS) files available at XMM ESAC webpages 11 All the images presented here are superpositions of the MOS1, MOS2, and pn images, obtained by using the emosaic SAS procedure. We produced vignetting-corrected count-rate maps. It is possible to correct for vignetting by dividing the superposed images by the corresponding superposed exposure maps produced by using the eexpmap SAS command. The pn exposure maps were scaled by the ratio of the pn/MOS effective areas to make MOS-equivalent superposed count-rate maps. We then smoothed the resulting count-rate maps adaptively in order to reach a user-defined signal-to-noise ratio by adopting the asmooth SAS task. We performed spatially resolved spectral analysis for all three EPIC detectors. For this purpose, we corrected the vignetting effect in the spectra using the evigweight SAS command. For each spectrum, we produced the redistribution matrix file (RMF, with the rmfgen task) and the ancillary response file (ARF, with the arfgen task) and we binned spectra to obtain at least 25 counts per bin.

We also analyzed the ROSAT All Sky Survey (RASS) data of the western region of Vela SNR to complement the XMM-Newton data analysis. The RASS was conducted using a Position-Sensitive Proportional Counter (PSPC) that operated in the 0.1-2.4 keV energy band. The RASS PSPC observation pointing the western part of Vela SNR was performed between October 17, 1990 and November 22, 1990, with pointing coordinate $\alpha_{J 2000}=8^{h} 15^{m} 00^{s}$ and $\delta_{J 2000}=-45^{\circ} 00^{\prime} 00^{\prime \prime}$ (Observation

\footnotetext{
https://www . cosmos. esa. int/web/xmm-newton/ filter-closed

http://xmm-tools. cosmos. esa.int/external/xmm calibration//background/bs_repository/blanksky_all. html
} 


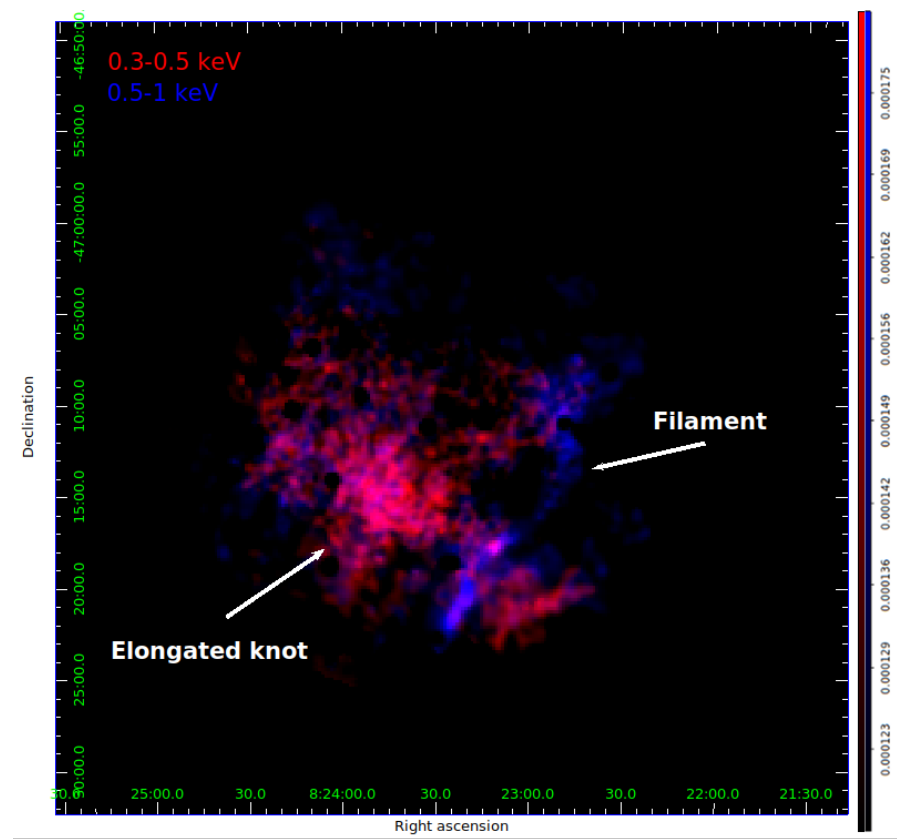

Fig. 2. EPIC count-rate color composite image in the $0.3-0.5 \mathrm{keV}$ band (red) and $0.5-1 \mathrm{keV}$ band (blue) in linear scale. The bin size is $10^{\prime \prime}$, and the image was adaptively smoothed to a signal-to-noise ratio of 20. North is up and east is to the left.

ID WG932517P_N1_SI01.N1) without any filter in survey mode and a total exposure of $23 \mathrm{ks}$. The total field of view (FoV) of the observation is a $6^{\circ} 30^{\prime} \times 6^{\circ} 30^{\prime}$ box.

The ROSAT archive provides processed event lists, distributed as FITS files, and no further data reduction is necessary for the user. We produced ROSAT count maps and spectra using the XSELECT analysis system tools extract image and extract spectrum, respectively. For the spectral analysis, we used the pspcc_gain1_256.rmf RMF file and pspcc_gain1_256.rsp on-axis response matrices file to calculate the ARF file for an off-axis region with the FTOOLS command pcarf. All these files are stored on an HEASARC ftp server ${ }^{2}$. We binned the spectra energy channels in order to have at least 25 counts per channel. The spectral analysis of XMMNewton and ROSAT spectra was performed with the HEASOFT software XSPEC version 12.9.1 (Arnaud 1996) with the solar abundances table from Anders \& Grevesse (1989).

\section{XMM-Newton results}

\subsection{Images}

In Fig. 2, we show the EPIC composite count-rate image of the Vela SNR knot K, showing the $0.3-0.5 \mathrm{keV}$ emission in red and the $0.5-1.0 \mathrm{keV}$ band emission in blue. In the $0.3-0.5$ $\mathrm{keV}$ band, an elongated knot clearly sticks out. This knot seems to be strongly elongated, extending for $\sim 20^{\prime}$ (corresponding to $\sim 5 \times 10^{18} \mathrm{~cm}$ at a distance of $280 \mathrm{pc}$ ) in the northeast-southwest direction, and only $\sim 5^{\prime}\left(\sim 1 \times 10^{18} \mathrm{~cm}\right)$ on the average in the southeast-northwest direction. The elongated knot is less visible in the $0.5-1 \mathrm{keV}$ band, where a narrow filament running in the northwest-southeast direction emerges.

In Fig. 3 we show the mosaic of images of the Vela SNR shrapnel $\mathrm{G}$ and knot $\mathrm{K}$ in the $0.3-0.6 \mathrm{keV}$ energy band (i.e., the

\footnotetext{
2 https://heasarc.gsfc.nasa.gov/FTP/caldb/data/rosat/ pspc/cpf/matrices/
}

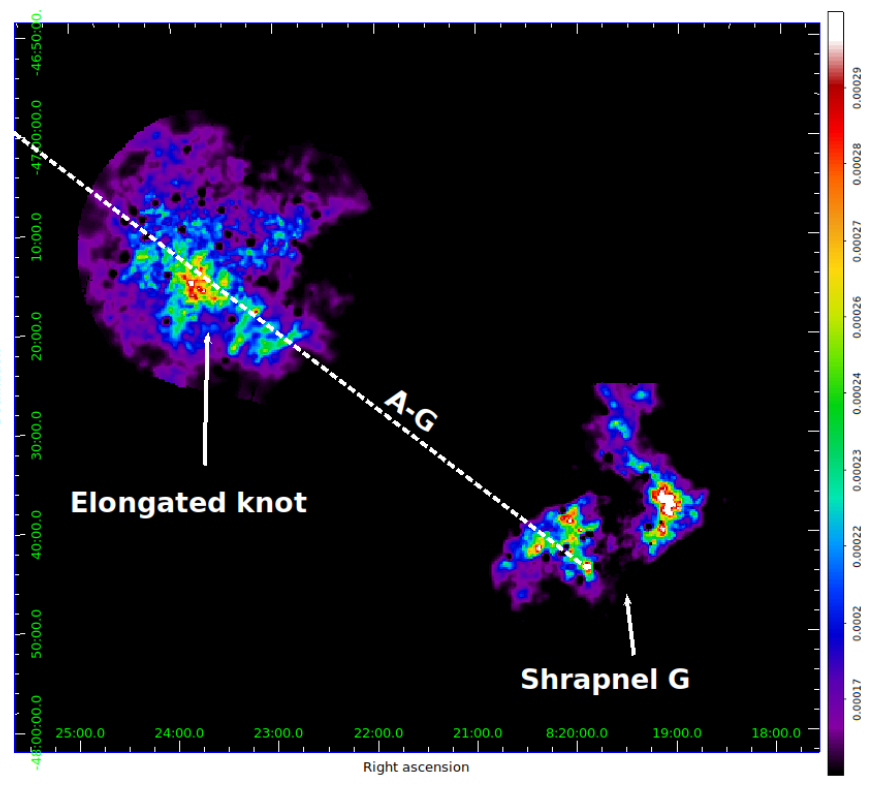

Fig. 3. EPIC count-rate image of shrapnel $\mathrm{G}$ and knot $\mathrm{K}$ in the $0.3-0.6$ $\mathrm{keV}$ energy band in squared scale. The bin size is $10^{\prime \prime}$ and the image was adaptively smoothed to a signal-to-noise ratio of 20 . The white dashed line connects shrapnel $\mathrm{G}$ and shrapnel A (shrapnel A is outside the field of view of this image, on the opposite side of Vela SNR, see Fig 1 .

soft energy band adopted by García et al. 2017) to reveal possible connections between the structures detected in the FoV of knot K and those observed in shrapnel G by García et al. (2017). The image clearly shows that the elongated knot remarkably lays along the line (indicated by a white dashed line in Fig. 3, see also Fig. 1) connecting shrapnel A, shrapnel G, and the explosion site. The explosion site, shown by the black cross in Fig. 1. can be estimated by taking into account the proper motion of the Vela pulsar (Caraveo et al. 2001) and assuming an age of 11000 yr. The excellent alignment between the explosion site, the elongated structure, and the two Si-rich shrapnel is suggestive of a possible physical link between the elongated knot and the Si-rich ejecta. This may indicate that the elongated knot is somehow part of a jet-like structure associated with shrapnel A and $\mathrm{G}$ (though its X-ray emission is much softer than that of the two shrapnel).

\subsection{Median photon energy map}

To investigate the thermal distribution of the X-ray emitting plasma, we produced maps of median photon energy $\left(E_{m}\right)$ for the pn camera, that is to say maps showing the median energy of the photons detected pixel-by-pixel in a given energy band. These maps not only provide information on the spatial distribution of the X-ray emission spectral hardness of the source, but also on the local value of the equivalent hydrogen column (the higher the absorption, the higher the $E_{m}$ value). The pixels in the map have a size of $20^{\prime \prime}$ so as to collect more than ten counts per pixel. We smoothed the map by adopting the procedure described in Miceli et al. (2008b), with $\sigma=60^{\prime \prime}$. We verified that the instrumental background does not affect the $E_{m}$ value, since it is from 15 up to 30 times lower than the signal. Also, we verified from the FWC file that there are no pieces of evidence of significant fluctuations across the FoV in pn instrumental background.

In Fig. 4 we show the pn $E_{m}$ map produced in the $0.3-1.0$ $\mathrm{keV}$ energy band. The map clearly shows that the $E_{m}$ of the elon- 


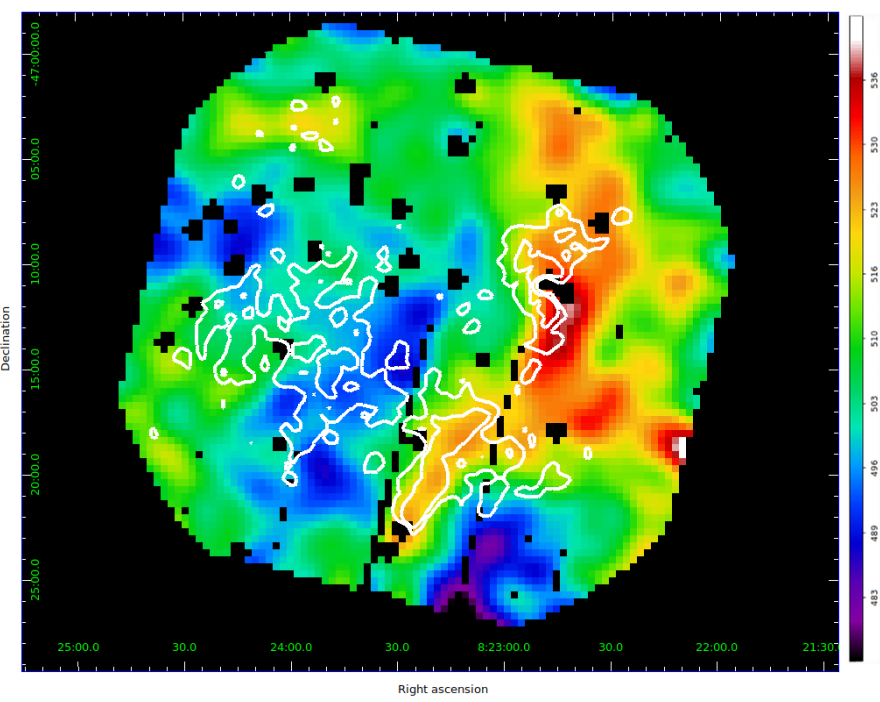

Fig. 4. Median photon energy map of the knot $\mathrm{K}$ region obtained with EPIC pn data in the $0.3-1.0 \mathrm{keV}$ energy band in linear scale. The map was smoothed by adopting a Gaussian kernel with $\sigma=60^{\prime \prime}$. The overlaid white contours indicate the count-rate levels at $50 \%$ and $60 \%$ of the maximum in the $0.5-1.0 \mathrm{keV}$ energy band. The bin size is $20^{\prime \prime}$ and the characteristic error for the median energy is $\sim 10 \mathrm{eV}$.

gated knot is lower than that of the filament. Since the interstellar absorption in this region of the Vela SNR is quite uniform (Lu \& Aschenbach 2000), the most natural explanation is that the plasma temperature is higher in the filament than in the elongated knot. This confirms that the two structures have different physical properties.

\subsection{Spectra}

The analysis described in the previous subsection allowed us to identify X-ray emitting plasma structures with homogeneous physical properties. To further investigate the physical and chemical properties of the plasma, we performed a spatiallyresolved spectral analysis. We first focused on the elongated knot which appears similar to a trailing wake of shrapnel G (see Fig. 3).

We selected a polygonal spectral-extraction region labeled Elong_knot, including the whole jet-like structure, indicated by the black polygon in Fig. 5 We extracted the spectrum of the background from the red region shown in Fig. 5, characterized by a very low surface brightness. By selecting other background regions (within the blue areas of Fig 5), we verified that the results of the spectral analysis do not change significantly: The best fit parameters are all consistent within $1 \sigma$. The spectra of all cameras above $1.3 \mathrm{keV}$ are dominated by the background, also including the Si fluorescence emission lines of the MOS instrumental background. We verified that the results of the spectral analysis do not change significantly by performing the analysis in the $0.3-2 \mathrm{keV}$ band or in the $0.3-1.3 \mathrm{keV}$ band. Thus we performed the analysis in the $0.3-1.3 \mathrm{keV}$ band to maximize the signal-to-noise ratio.

The elongated knot spectrum shows thermal features, namely emission line complexes of O VII at $\sim 0.57 \mathrm{keV}$ and of $\mathrm{Ne}$ IX at $\sim 0.92 \mathrm{keV}$ (see Fig. 6). We fit the spectra with a model of isothermal optically-thin plasma in collisional ionization equilibrium (CIE) (vapec model in XSPEC) with nonsolar abundances. We also included the effects of photoelectric absorption by ISM (tbabs model in XSPEC). By letting the $\mathrm{O}$ abundance

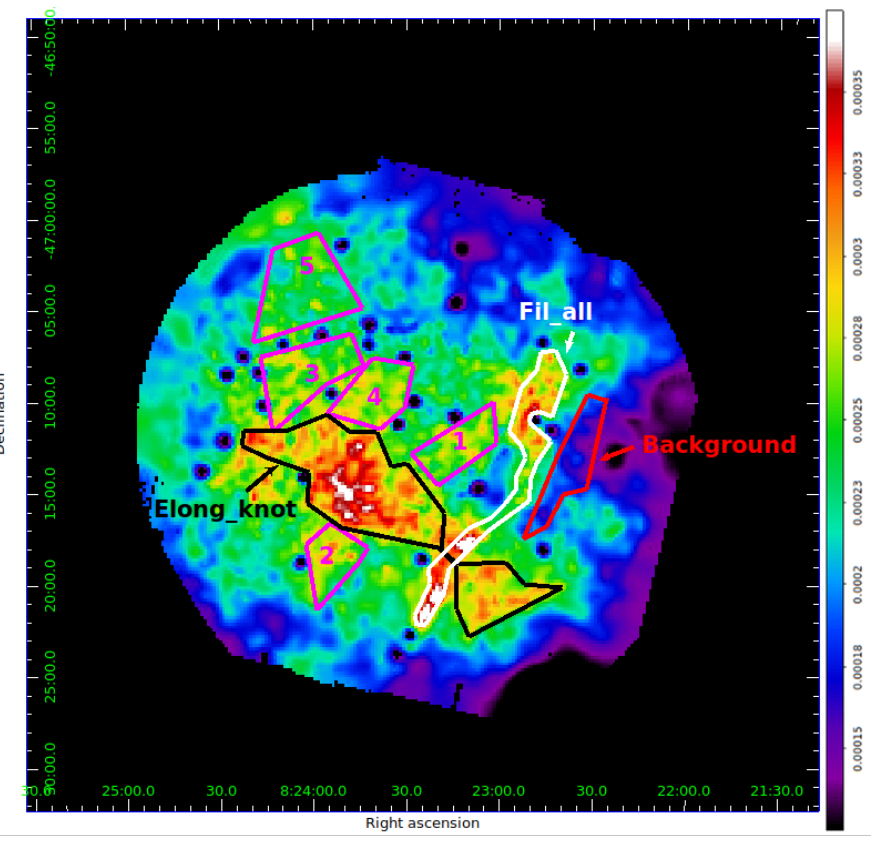

Fig. 5. EPIC count-rate image in the $0.3-1.0 \mathrm{keV}$ energy band in linear scale. The bin size is $10^{\prime \prime}$ and the image was adaptively smoothed to a signal-to-noise ratio of 20 . Regions selected to extract the spectra of the elongated knot (black polygon) and of the filament (white polygon) are superimposed. The region selected for background extraction is shown in red. The regions selected to extract control spectra are shown in magenta (see Sect. 3.3 .

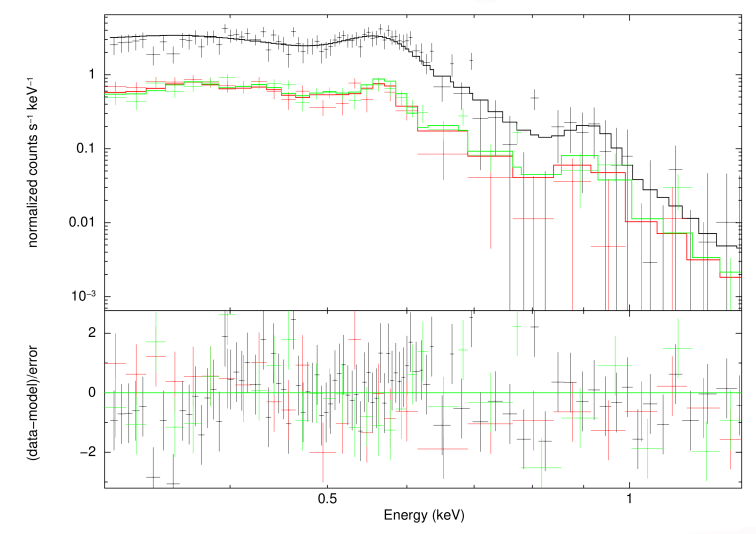

Fig. 6. EPIC spectra (pn upper, MOS1,2 lower) extracted from the Elong_knot region (shown in Fig. 5 with the corresponding CIE best-fit model and residuals in the $0.3-1.3 \mathrm{keV}$ band.

free to vary, the fit provides $\chi^{2} /$ d.o.f. $=328.14 / 300$. We verified that the quality of the fit does not significantly improve by letting the Ne abundance free to vary. Moreover, the fitting procedure is not sensitive to the $\mathrm{Fe}$ abundance, probably because the plasma temperature $\left(T \sim 1.5 \times 10^{6} \mathrm{~K}\right)$ is too low for significant Fe L lines to be emitted in the $0.7-1.3 \mathrm{keV}$ energy band. Best-fit values are shown in Tab. 1 Error bars are at a $90 \%$ confidence level.

The best fit temperature is somehow entangled with the best fit value of the column density (as shown in Fig. 7), which is forced to be lower than $6 \times 10^{20} \mathrm{~cm}^{-2}$, in agreement with the findings by Lu \& Aschenbach (2000) in this region of the shell. 
Table 1. Best-fit parameters for the region Elong_knot shown in Fig. 5 with the CIE best-fit model (vapec).

\begin{tabular}{cc}
\hline \hline Parameter & Elong_knot \\
\hline${ }^{a} N_{\mathrm{H}}\left(10^{22} \mathrm{~cm}^{-2}\right)$ & $>0.05$ \\
$k T(\mathrm{keV})$ & $0.124_{-0.005}^{+0.006}$ \\
$\mathrm{O} / \mathrm{O}_{\odot}$ & $0.36_{-0.05}^{+0.07}$ \\
$\mathrm{Ne} / \mathrm{Ne}_{\odot}$ & $1^{b}$ \\
${ }^{c} n^{2} l\left(10^{18} \mathrm{~cm}^{-5}\right)$ & $1.7_{-0.2}^{+0.1}$ \\
$\chi^{2} /$ d.o.f. & $327.97 / 300$ \\
\hline
\end{tabular}

Errors are at the $90 \%$ confidence level.

Notes. ${ }^{a}$ Upper limit fixed to $0.06 .{ }^{b}$ Fixed value. ${ }^{c}$ Emission measure per unit area.

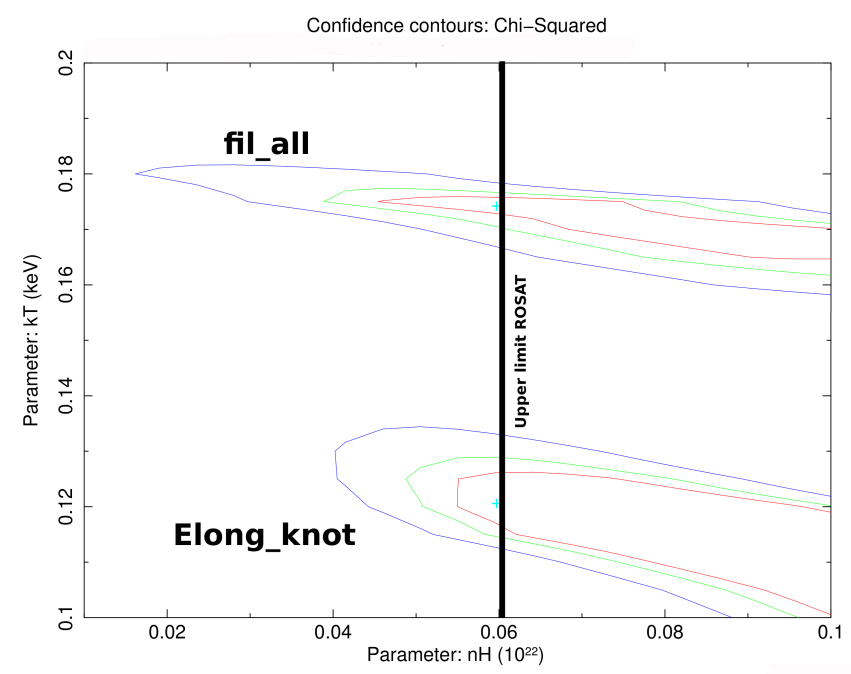

Fig. 7. $68 \%$ (red), $95 \%$ (green), and $99.5 \%$ (blue) contour levels of the $N_{\mathrm{H}}$ vs. $k T$ best fit values, as derived from the spectral analysis of the fil_all and Elong_knot spectra. The black line shows the upper limit for $N_{\mathrm{H}}$ found by Lu \& Aschenbach (2000).

Though the spectral analysis of shrapnel G makes it possible to constrain the chemical abundances of more elements than that of knot $\mathrm{K}$, the $\mathrm{Ne}$ (and $\mathrm{Mg}$ ) to $\mathrm{O}$ abundance ratio that we found in the elongated knot $\left(\mathrm{Ne} / \mathrm{O}=\mathrm{Mg} / \mathrm{O}=2.6_{-0.4}^{+0.5}\right.$ with $\mathrm{Ne}$ and $\mathrm{Mg}$ abundances fixed to the solar values) is similar to that found in the ejecta in other regions of the Vela SNR. This includes shrapnel G (García et al.2017), shrapnel A (Katsuda \& Tsunemi 2006), shrapnel D (Katsuda \& Tsunemi 2005), the ejecta found in the RegNe region (Miceli et al. 2008a), and those in the Vela $\mathrm{X}$ region (LaMassa et al. 2008). Though an ISM origin cannot be firmly excluded, this result seems to support an ejecta origin for the elongated knot.

We also extracted spectra from five regions surrounding the Elong_knot (labeled reg 1-5 in Fig. 5) and found that the plasma temperature therein is always significantly lower than that of the knot. Moreover, the $\mathrm{Ne} / \mathrm{O}$ abundance ratio is systematically (sometimes significantly) lower than that of the elongated knot (see Tab. 2). These results clearly show that all these regions do not belong to the elongated knot, which is indeed confined to a narrow, jet-like stripe along the direction connecting shrapnel A and shrapnel $\mathrm{G}$.

García et al. (2017) modeled the spectrum of shrapnel $\mathrm{G}$ with an absorbed isothermal component in nonequilibrium of ion-
Table 2. Best-fit parameters for spectra from regions labeled from 1 to 5 shown in Fig. 5

\begin{tabular}{cccccc}
\hline \hline Parameter & 1 & 2 & 3 & 4 & 5 \\
\hline $\mathrm{kT}(\mathrm{keV})$ & $0.108_{-0.012}^{+0.009}$ & $0.109_{-0.015}^{+0.005}$ & $0.108_{-0.015}^{+0.004}$ & $0.109_{-0.009}^{+0.007}$ & $0.108_{-0.017}^{+0.003}$ \\
$\mathrm{O} / \mathrm{O}_{\odot}$ & $0.40_{-0.1}^{+0.34800}$ & $0.66_{-0.16}^{+0.19}$ & $0.5_{-0.1}^{+0.2}$ & $0.56_{-0.15}^{+0.15}$ & $0.6_{-0.1}^{+0.4}$ \\
$n^{2} l\left(10^{18} \mathrm{~cm}^{-5}\right)$ & $1.4_{-0.1}^{+0.5}$ & $2.3_{-0.4}^{+0.6}$ & $1.5_{-0.2}^{+0.6}$ & $1.6_{-0.2}^{+0.3}$ & $1.3_{-0.1}^{+0.6}$ \\
$\chi^{2}$ d.o.f. & $255.45 / 225$ & $175.15 / 168$ & $260.62 / 252$ & $284.48 / 239$ & $267.15 / 243$ \\
\hline
\end{tabular}

Errors are at the $90 \%$ confidence level.

Notes. $N_{\mathrm{H}}$ value fixed to 0.06 and $\mathrm{Ne} / \mathrm{Ne}_{\odot}$ value is fixed to 1 .

ization (NEI) and nonsolar abundances. We checked if a similar scenario can be adopted for the elongated knot $\mathrm{K}$ by fitting its spectrum with the same model as that in García et al. (2017) by letting only the plasma temperature and the $n_{H}$ free to vary. We thus obtained a good fit to the Elong_knot spectrum $\left(\chi^{2} /\right.$ d.o.f. $\left.=435.55 / 302\right)$. Nevertheless, the quality of the fit is clearly worse than that obtained by the model in CIE. In any case, we found that the two spectral models adopted (CIE and NEI) clearly show that the plasma temperature of the elongated knot is significantly lower than that of shrapnel G (see Tab. 1 and Tab. 3), as we expected. Because of its interaction with the medium swept, the head of a jet-like structure (shrapnel G) is hotter than its tail (knot $\mathrm{K})$, that is interacting with an expanding medium.

At the low temperature of the elongated knot, the emissivity of the Si XIII emission line is extremely low and this hampers the emergence of the Si emission line above the continuum component of the spectrum and above the background. This hinders the possibility of obtaining an accurate measure of the Si abundance in the elongated knot, given that the emerging spectrum is actually insensitive to this parameter. We have repeated the spectral analysis of the elongated knot either in CIE or in NEI by also including the spectral data points in the $1.3-2 \mathrm{keV}$ energy band to include the energy bins corresponding to the Si XIII emission line (around $1.8 \mathrm{keV}$ ). We let the $\mathrm{Si}$ abundance free to vary in the fitting procedure. We found that the Si abundance is almost unconstrained (as expected), obtaining a best fit value of $6 \pm 4$ in NEI, and the abundance constrained to be $<2$ in CIE. In any case, the $\mathrm{Si}$ abundance is consistent with that found in shrapnel $G$ by García et al. (2017) and in shrapnel A by Katsuda \& Tsunemi (2006). Therefore, although it is not possible to prove that the jet-like structure is Si-rich, the spectral analysis shows that a spectrum of Si-rich plasma is perfectly consistent with the observed spectrum and that the jet-like structure could have the same Si abundance as shrapnel A and shrapnel G.

The second X-ray emitting structure that emerges in Fig. 2 has the shape of a narrow filament. Therefore, we extracted the spectrum from a large region indicated by the white polygon, labeled fil_all, in Fig. 5, including the whole filament. We adopted the same background spectrum as that used for the elongated knot. The spectrum of the region shows thermal emission, characterized by emission lines from $\mathrm{O}$ VII at $\sim 0.57 \mathrm{keV}$, from $\mathrm{O}$ VIII at $\sim 0.65 \mathrm{keV}$, and from Ne IX at $\sim 0.92 \mathrm{keV}$ (see Fig. 8). To fit the spectrum, we first adopted a model of isothermal plasma in CIE (vapec) with solar abundances, including the effects of photoelectric absorption by the ISM (tbabs). We fixed the $N_{\mathrm{H}}$ to the best-fit value found for the elongated knot. By letting the $\mathrm{O}$ and $\mathrm{Ne}$ abundance free to vary, the fit provides $\chi^{2} /$ d.o.f. $=337.67 / 276$. Best-fit values are shown in Tab. $4 . \mathrm{Er}-$ ror bars are at a $90 \%$ confidence level. EPIC spectra with the corresponding best-fit model and residuals are shown in Fig. 
Table 3. Best-fit parameters for region Elong_knot shown in Fig. 5 obtained with the NEI model compared with those found in shrapnel $\mathrm{G}$ by García et al. (2017).

\begin{tabular}{ccc}
\hline \hline Parameter & Elong_knot & Shrapnel G \\
\hline$N_{\mathrm{H}}\left(10^{22} \mathrm{~cm}^{-2}\right)$ & $0.06^{\star}$ & $0.022_{-0.014}^{+0.014}$ \\
$k T(\mathrm{keV})$ & $0.113_{-0.006}^{+0.011}$ & $0.49_{-0.04}^{+0.04}$ \\
$\tau\left(10^{10} \mathrm{~s} \mathrm{~cm}^{-3}\right)$ & $3.1^{\star}$ & $3.1_{-0.3}^{+0.3}$ \\
$\mathrm{C} / \mathrm{C}_{\odot}=N / N_{\odot}=O / O_{\odot}$ & $0.47^{\star}$ & $0.47_{-0.10}^{+0.10}$ \\
$\mathrm{Ne} / \mathrm{Ne} \odot$ & $1.33^{\star}$ & $1.3_{-0.2}^{+0.2}$ \\
$\mathrm{Mg} / \mathrm{Mg} \odot$ & $0.92^{\star}$ & $0.9_{-0.2}^{+0.2}$ \\
$\mathrm{Si} / \mathrm{Si} \odot$ & $2.24^{\star}$ & $2.2_{-0.9}^{+0.9}$ \\
$\mathrm{Fe} / \mathrm{Fe} \odot$ & $0.29^{\star}$ & $0.29_{-0.08}^{+0.08}$ \\
$n^{2} l\left(10^{18} \mathrm{~cm}^{-5}\right)$ & $1.7_{-0.2}^{+0.1}$ & $0.21_{-0.06}^{+0.06}$ \\
$\chi^{2} /$ d.o.f. & $435.55 / 302$ & $635.91 / 451$
\end{tabular}

Errors are at a $90 \%$ confidence level.

Notes. ${ }^{\star}$ Fixed value.

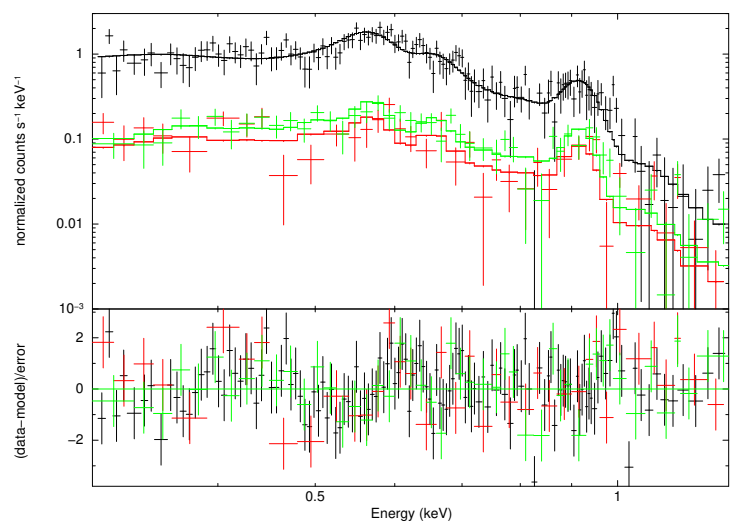

Fig. 8. EPIC spectra (pn upper, MOS1,2 lower) extracted from the fil_all region (shown in Fig. 5) with the corresponding CIE best-fit model and residuals in the $0.3-1.3 \mathrm{keV}$ band.

8. The best-fit temperature in the fil_all region is significantly higher than that of the elongated knot (see Fig. 7. Tab. 1 and Tab. 4). This confirms that the filament is indeed hotter than the elongated knot, as suggested by the image analysis. The $\mathrm{Ne} / \mathrm{O}$ ratio is significantly higher than that found for the knot $\left(\mathrm{Ne} / \mathrm{O}_{\text {fil_all }}=4.0_{-0.8}^{+0.6}\right.$ versus Ne/O $\left.\mathrm{O}_{\text {Elong_knot }}=2.6_{-0.5}^{+0.4}\right)$, and it is also higher than that in the previously mentioned ejecta fragments. The different temperatures and abundances confirm the different nature of the two plasma structures.

\section{ROSAT results}

\subsection{Images}

The filament that we found in the XMM-Newton observation runs from the upper right to the lower left corners of the instrument FoV (see the blue structure in Fig. 2) and it may therefore be a part of a large-scale structure extending beyond the $X M M$ Newton FoV. We thus investigated the nature of this feature by analyzing RASS observations covering the whole western part of Vela SNR.

Article number, page 6 of 10
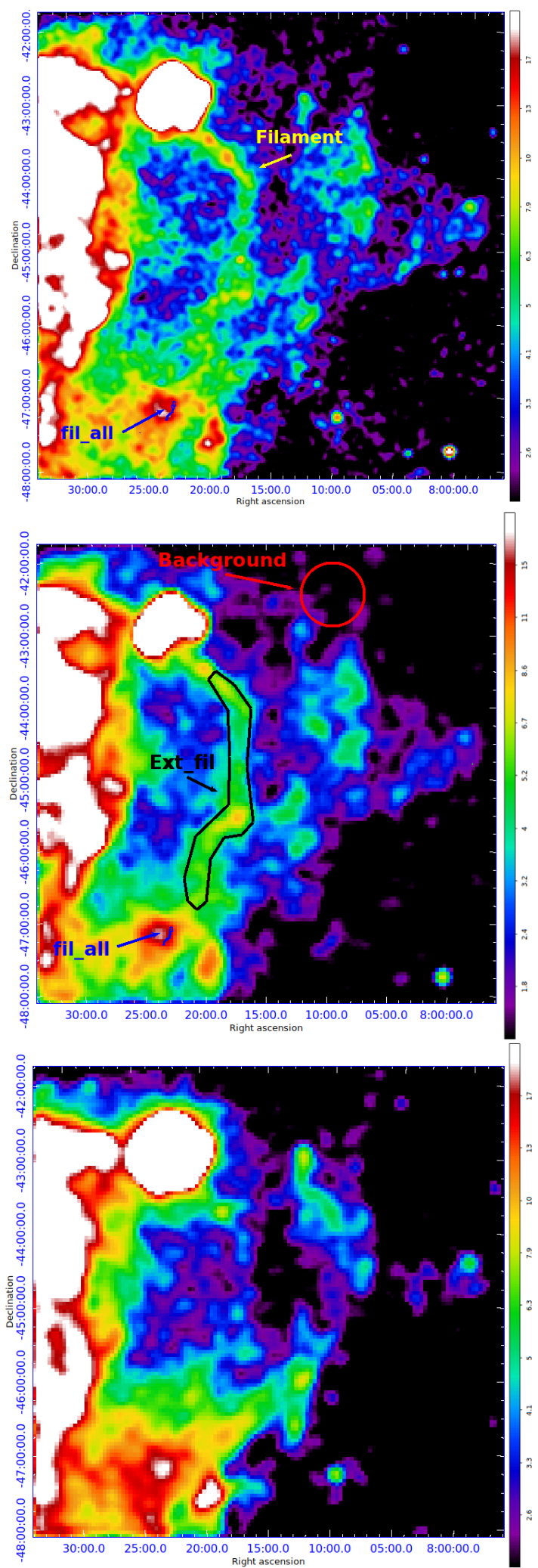

Fig. 9. Top panel: ROSAT PSPC map of photon counts in the $0.1-2.4$ $\mathrm{keV}$ energy band in squared root scale. The bin size is $1^{\prime}$. The map was smoothed through the convolution with a Gaussian with $\sigma=3^{\prime}$ (3 pixel). The fil_all spectral region (see Fig. 5) is superimposed in blue. Center panel: ROSAT PSPC map of photon counts in the $0.3-0.5 \mathrm{keV}$ energy band in squared root scale, smoothed with $\sigma=9^{\prime}$. The bin size is $3^{\prime}$. The red circle indicates the background spectrum region, and the black polygon is the filament spectrum extraction region. The fil_all spectral region is superimposed in blue. Bottom panel: ROSAT PSPC map of photon counts in the $0.5-1.0 \mathrm{keV}$ energy band in squared root scale, smoothed with $\sigma=9^{\prime}$. The bin size is $3^{\prime}$. 
Table 4. Filament spectra best-fit parameters with the CIE best-fit model (vapec).

\begin{tabular}{cc}
\hline \hline Parameter & fil_all \\
\hline $\mathrm{kT}(\mathrm{keV})$ & $0.174_{-0.002}^{+0.002}$ \\
$\mathrm{O} / \mathrm{O}_{\odot}$ & $0.48_{-0.06}^{+0.07}$ \\
$\mathrm{Ne} / \mathrm{Ne} \odot$ & $1.9_{-0.3}^{+0.4}$ \\
$n^{2} l\left(10^{18} \mathrm{~cm}^{-5}\right)$ & $0.93_{-0.12}^{+0.12}$ \\
$\chi^{2} /$ d.o.f. & $337.67 / 276$ \\
\hline
\end{tabular}

Errors are at a $90 \%$ confidence level.

In Fig. 9 we show maps of photon counts of the western part of Vela SNR in the broad ROSAT bandpass (i.e., $0.1-2.4 \mathrm{keV}$ ) and in the $0.3-0.5 \mathrm{keV}$ (soft) and $0.5-1.0 \mathrm{keV}$ (hard) energy bands. In the ROSAT broad and soft band images, an extended filament running from north (at a position approximately corresponding to the projected location of the Puppis A SNR) to the south, and extending down exactly to the knot $\mathrm{K}$ region, is clearly visible. The extended filament blends with the surrounding emission in the $0.5-1.0 \mathrm{keV}$ image.

\subsection{Median photon energy maps}

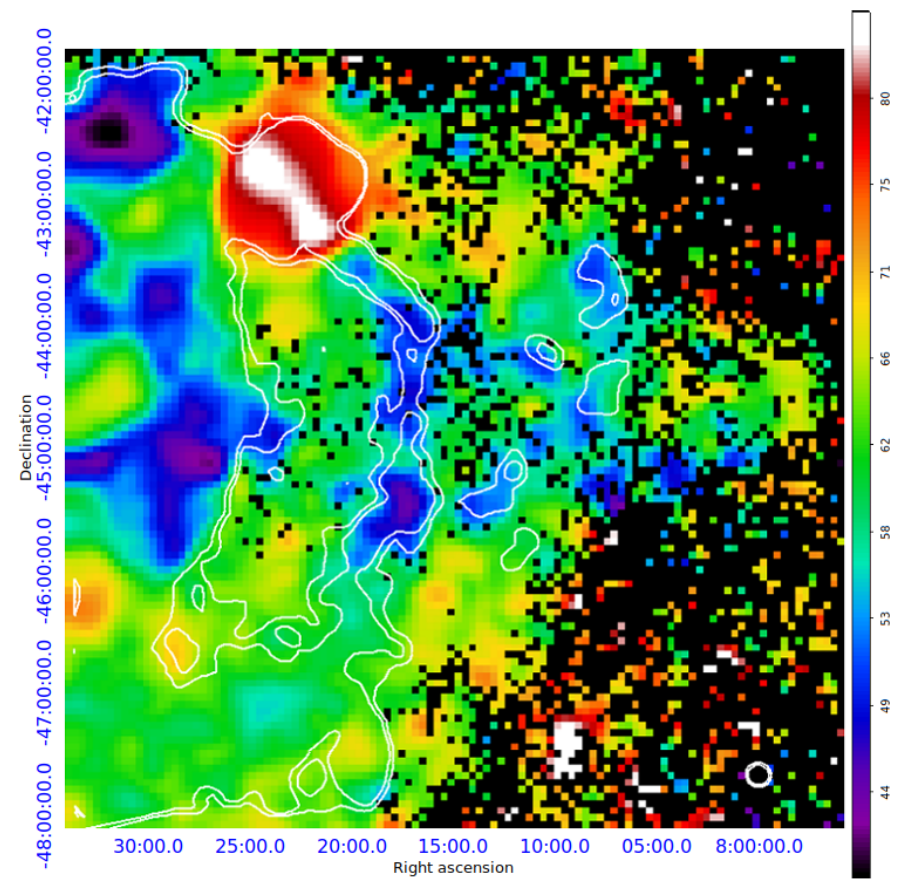

Fig. 10. Median photon energy map of the western part of the Vela shell in the $0.3-1.3 \mathrm{keV}$ energy band with a bin size of $3.5^{\prime}$ in linear scale. The scale is in units of $10 \mathrm{eV}$. White contours mark the photons'. count number levels between 4 and 5 counts in the $0.3-0.5 \mathrm{keV}$ energy band.

In Fig. 10 we show the $E_{m}$ map of the ROSAT RASS observation in the $0.3-1.3 \mathrm{keV}$ energy band that we produced to further characterize the $E_{m}$ of the extended filament. The image shows that the X-ray emission of the extended filament is less energetic than that of the surrounding plasma. Also, the whole structure is coherent in $E_{m}$ on a very large spatial scale, comparable with the diameter of the shell. The relatively low $E_{m}$ of the extended filament $(450-550 \mathrm{eV}$, indicated by the blueish structure in Fig. 10 presents only minor spatial variations that may
Table 5. ROSAT filament best-fit.

\begin{tabular}{cc}
\hline \hline parameter & Ext_fil \\
\hline$N_{\mathrm{H}}\left(10^{22} \mathrm{~cm}^{-2}\right)$ & $0.029_{-0.004}^{+0.006}$ \\
$k T(\mathrm{keV})$ & $0.177_{-0.016}^{+0.011}$ \\
$\mathrm{O} / \mathrm{O}_{\odot}$ & $0.22_{-0.08}^{+0.09}$ \\
$\mathrm{Ne} / \mathrm{Ne} \odot$ & $<1.5$ \\
$n^{2} l\left(10^{18} \mathrm{~cm}^{-5}\right)$ & $0.017_{-0.005}^{+0.005}$ \\
$\chi^{2} /$ d.o.f & $93.15 / 94$ \\
\hline
\end{tabular}

The abundance of $\mathrm{O}$ was frozen to the best-fit parameter in order to reduce the uncertainty of the kT parameter. The abundance of $\mathrm{O}$ was determined by freezing $\mathrm{Ne}$ abundance. Errors are at a $90 \%$ confidence level.

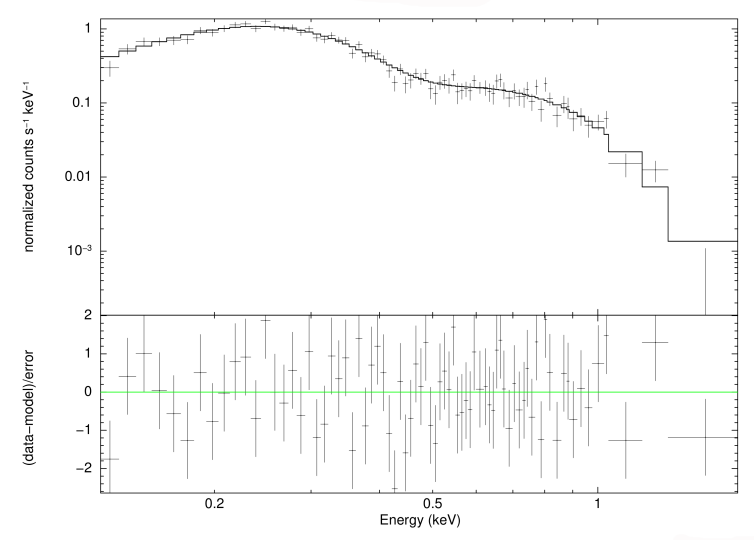

Fig. 11. ROSAT PSPC-C spectrum of region Ext_fil (black polygon in Fig. 9. center panel) with the best-fit CIE model in the $0.3-1.8 \mathrm{keV}$ energy band. The bottom panel shows the residual between the data and the model.

not necessarily be caused by inhomogeneities in the plasma temperature. Variations in $E_{m}$ of the soft energy band considered, in fact, may be associated with different values of the absorbing column density, which is expected to vary on these large spatial scales (Lu \& Aschenbach 2000).

\subsection{Spectra}

The ROSAT image analysis suggests that the narrow filament detected in the XMM-Newton observation may be a portion of a much larger structure that clearly sticks out in Fig. 9. We extracted the spectrum from the region indicated by the black polygon, labeled Ext_fil, in Fig. 9, we also extracted the background spectrum from the circular region indicated in red in Fig. 9 . We fit the spectrum by adopting the best-fit model that we found for the XMM-Newton filament, that is CIE thermal emission from an isothermal plasma (vapec) with $\mathrm{O}$ and $\mathrm{Ne}$ abundances free to vary, and including the effects of ISM absorption (tbabs). Despite the poor ROSAT PSPC energy resolution, we were able to derive the best-fit parameters independently from the XMMNewton spectral analysis of the filament (see Tab. 5). The PSPC spectrum of Ext_fil with the corresponding best-fit model and residual is shown in Fig. 11 .

The best-fit temperature of the large filament is remarkably consistent with that obtained for the narrow filament in the 


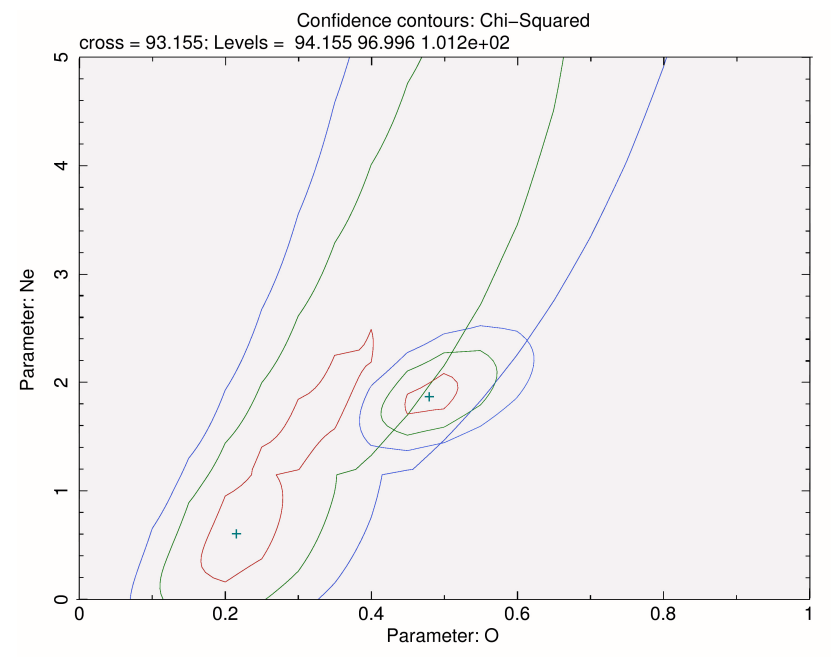

Fig. 12. $68 \%$ (red), $95 \%$ (green), and $99.5 \%$ (blue) contour levels of the Ne abundance vs. O derived from the spectral analysis of the Ext_fil spectrum with the same contour levels of fil_all spectrum superimposed.

XMM-Newton observation $\left(k T_{\text {fil_all }}=0.174_{-0.002}^{+0.002} k T_{E x t \_f i l}=\right.$ $0.177_{-0.016}^{+0.011}$, see also Tab. 4 and Tab. 5 for comparison). It is important to note that $\mathrm{Ne}$ and $\mathrm{O}$ abundances are not well constrained because of the low energy resolution of the PSPC-C ROSAT spectra. Fig. 12 shows the $68 \%, 95 \%$, and $99.5 \%$ confidence contour levels of the $\mathrm{Ne}$ abundance versus the $\mathrm{O}$ abundance derived from the ROSAT spectrum of the large filament compared to the levels for the narrow filament in the $X M M$ Newton data. The plot shows that abundances are consistent within the $2 \sigma$ (green) confidence level, thus confirming the homogeneous chemical composition of the filament. These results strongly indicate that the narrow filament is part of a coherent, bent, giant structure, running north-south behind the eastern rim of the Vela shell.

\section{Discussions}

In this paper, we have presented a detailed study of an XMMNewton observation of a bright X-ray emitting clump, namely knot K, located behind shrapnel $\mathrm{G}$ in the southwestern region of Vela SNR (see Fig. 1). By analyzing the XMM-Newton observation, we found an X-ray emitting plasma structure (predominantly in the $0.3-0.5 \mathrm{keV}$ energy band, Fig. 2), which was remarkably elongated in the direction connecting shrapnel $\mathrm{A}$ and shrapnel G (see Fig. 3p (i.e., the only two Si-rich shrapnel detected in the Vela SNR so far). Furthermore, the elongated knot points toward the explosion site of the Vela SNR. This structure shows a soft thermal spectrum with nonsolar abundances, as shown in Fig. 6, and a temperature significantly lower than that of shrapnel A and G. Although the elongated knot has a low temperature that hampers the detection of over-solar Si abundance, we found that the $\mathrm{Ne}$ to $\mathrm{O}$ abundance ratio is consistent with that of shrapnel A (Katsuda \& Tsunemi 2006) and shrapnel G (García et al.|2017). Moreover, the Si abundance we found (though poorly constrained) is consistent with that observed in those ejecta fragments. Enhanced $\mathrm{Ne} / \mathrm{O}$ abundance ratios have also been observed in ISM clumps within the Vela SNR (Miceli et al. 2005. Katsuda et al. 2011), though with slightly lower values than those presented in Table 1. It is then possible that the elongated structure is a shocked ISM cloudlet. However, its highly elongated morphology seems to suggest an association with shrapnel G. Moreover, its chemical composition is consistent with being the same as that of shrapnel A and shrapnel G. In summary, our results show that a physical relationship between the jet-like elongated knot and the two Si-rich shrapnel is likely.

Assuming that the elongated knot has a cylindrical symmetry, we calculated the volume $(V)$ of the X-ray emitting structure through the relation $V=\pi \frac{D^{2}}{4} l$ where $l$ is the projected length of the elongated knot $\left(l \sim 5 \times 10^{18} \mathrm{~cm}\right)$ and $D$ is its projected thickness $\left(D \sim 1 \times 10^{18} \mathrm{~cm}\right)$, thus obtaining $V \sim 4 \times 10^{54} \mathrm{~cm}^{3}$. We point out that from the X-ray image, we can only measure the projected size of the features in the plane of the sky, so this value should be considered as a lower limit. However, since the structure is close to the border of the shell, the actual value may not differ too much from the one reported here. Through the best-fit value of the plasma emission measure and volume, we estimated a number density of $\bar{n}=1.20_{-0.07}^{+0.05} \mathrm{~cm}^{-3}$ for the elongated knot and a total mass of $M=1.00_{-0.04}^{+0.06} \times 10^{31} \mathrm{~g}\left(\sim 0.005 M_{\odot}\right)$, using as average atomic mass $\mu=2.1 \times 10^{-24} \mathrm{~g}$ (value for solar abundances). However this value is an upper limit since we expect oversolar chemical abundances 3

Considering the projected distance between the explosion center of Vela SNR and the elongated knot and the age of the remnant $\left(\simeq 11000\right.$ yrs, Taylor et al. 1993), we obtained $\mathrm{v}_{k} \sim 1.2 \times$ $10^{8} \mathrm{~cm} / \mathrm{s}(1200 \mathrm{~km} / \mathrm{s})$ and a kinetic energy of $E_{k}=7.2_{-0.4}^{+0.3} \times 10^{46}$ erg. However, if we take into account that the speed of the elongated knot decreases with time and accounts only for the projected velocity, this value of kinetic energy should be considered as a lower limit. Values of mass and kinetic energy found for the elongated knot are very similar to those found for shrapnel $\mathrm{G}$ by García et al. (2017). By assuming that the elongated knot is part of a jet-counterjet-like structure with shrapnel $A$ and $G$, its total mass and kinetic energy are $M=0.018 M_{\odot}$ and $E=4.7 \times 10^{47}$ erg, respectively.

Similar jet-like features have been observed in a handful of core-collapse SNRs and may be associated with anisotropies in the SN explosion (Miceli et al. 2013, Tsebrenko \& Soker 2015. Orlando et al. 2016, Bear \& Soker 2018, Tutone et al. 2020. ). Miceli et al. (2008b) found an indication of a jet-like structure for the galactic SNR W49B with mass $M=6 M_{\odot}$ and $E=1.2 \times 10^{50}$ (see also Lopez et al.|2013). However, it has been suggested that this collimated structure may not be intrinsic and may result from a (nearly) spherical explosion in a barrel-like circumstellar environment (Miceli et al.2008b, Zhou et al.2011. Zhou \& Vink 2018). Also the Cas A SNR, deeply analyzed by Hwang \& Laming (2012), shows a jet of Si-rich plasma. Laming et al. (2006) suggest that its origin lies in an explosive jet. Detailed 3-D hydrodynamic simulations by Orlando et al. (2016) proved that this jet can be explained as the result of velocity and density inhomogeneities in the ejecta profile of the exploding star. According to these simulations, the mass and kinetic energy of the jet-counterjet structure in Cas A are $M=0.05 M_{\odot}$ and $E=5.2 \times 10^{49} \mathrm{erg}$, respectively. Another jet-like feature in the ejecta has been recently discovered in the SNR IC 443 by Greco et al. (2018) who identified a jet-like structure with $\mathrm{Mg}$-rich plasma in overionization with mass and kinetic energy $M \sim 0.03 M_{\odot}$ and $E \sim 3 \times 10^{48} \mathrm{erg}$, respectively. A comparison between the Vela SNR and the other jet-like structures observed in core-collapse SNRs shows a wide range of masses and energies, as shown in Fig. 13. A simple linear regression gives $E=(4.7 \pm 1.7) \times 10^{49} \mathrm{erg} \cdot M / M_{\odot}$, but large residuals are present

\footnotetext{
3 The moderate spectral resolution of CCD spectrometers hampers the possibility of measuring absolute abundances and only reliable estimates of relative abundances can be obtained (see Greco et al. 2020).
} 


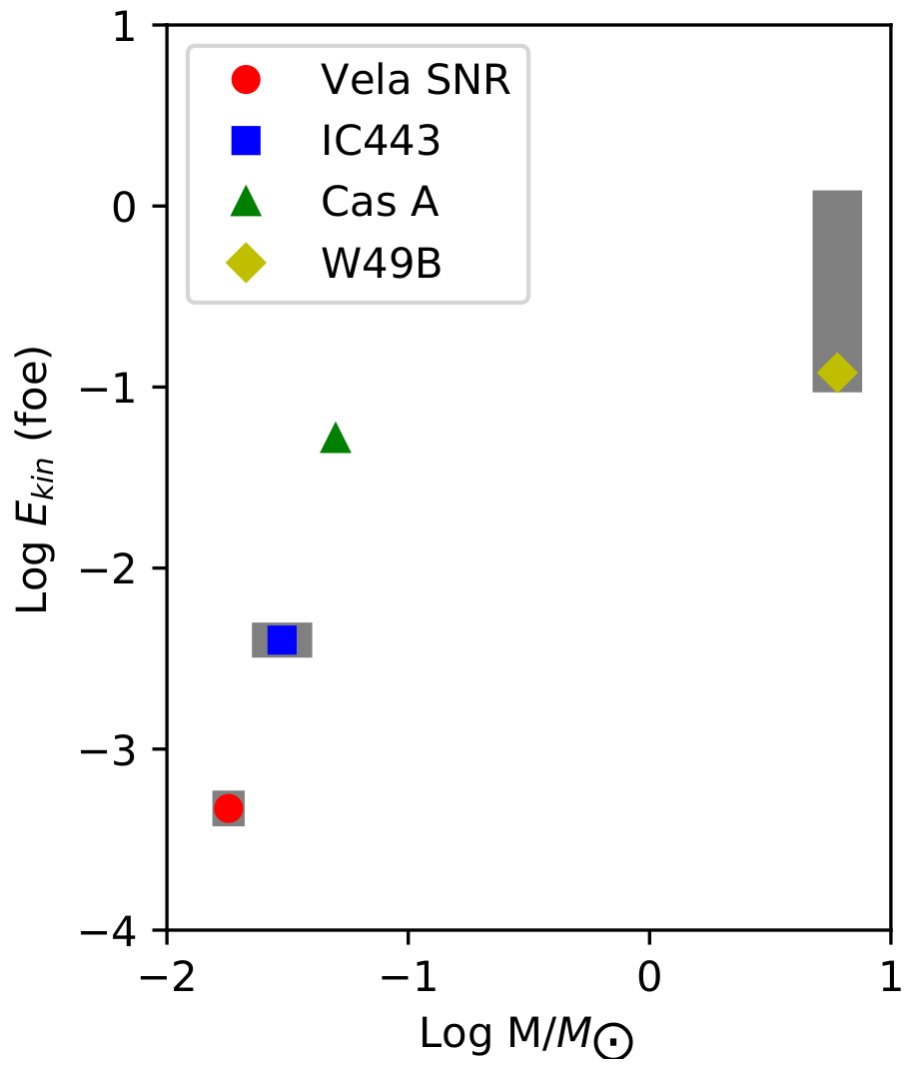

Fig. 13. Kinetic energy vs. mass of the jet-like structure observed so far in core collapse SNRs. Kinetic energy is expressed in foe $\left(1 \mathrm{foe}=10^{51}\right.$ erg). Values are from this paper, Katsuda \& Tsunemi (2006) and García et al. (2017) (Vela SNR), Greco et al. (2018) (IC443), Orlando et al. (2016) (Cas A), and Miceli et al. (2008b) (W49B).

and the number of data points is too limited to get robust information. We conclude that the morphology, the position, and the spectral analysis strongly suggest that the elongated knot is part of a knotty, collimated ejecta structure and/or an ejecta trailing wake left behind the supersonic motion of shrapnel G.

The $0.5-1.0 \mathrm{keV}$ count-rate image of the XMM-Newton observation dominantly shows the emission of a narrow filamentary plasma structure (see Fig. 2). The filament has a thermal spectrum (Fig. 8) and its temperature is significantly higher than that of the elongated knot and uniform along its whole length (see Fig. 4). The $\mathrm{Ne}$ to $\mathrm{O}$ ratio $\left(\mathrm{Ne} / \mathrm{O}=4 \cdot 0_{-0.8}^{+0.6}\right)$ is significantly higher than that of the elongated knot and, in general, than that of the ejecta fragments of Vela SNR. This indicates that the filamentary structure may have a different nature.

We demonstrated, thanks to the RASS data of Vela SNR, that this X-ray emitting plasma structure is the southern edge of a more extended filament that runs from the northern rim of the shell to knot K (see Fig. 9). The extended filament is a giant Xray emitting structure with a uniform temperature, whose physical and chemical properties are consistent with those measured in the XMM-Newton filament (as shown in Tab. 4, Tab. 5, and Fig. 12). The projected length of this extremely long structure is $l \sim 6 \times 10^{19} \mathrm{~cm}$. The distance between the explosion site and the extended filament shows relatively small variations, ranging from $\sim 4.5 \times 10^{19} \mathrm{~cm}$ to $\sim 5.5 \times 10^{19} \mathrm{~cm}$.

As shown with the ROSAT results, this long filament is located well behind the border of the shell and its X-ray emission is softer than that of the forward shock (see Fig. 10). Soft emission may be the result of an interaction with a dense en- vironment, given that the shock speed scales as the inverse of the square root of the particle density and the post-shock temperature increases as the square of the shock speed. This indication, together with the almost circular shape, centered at the position of the SN progenitor, suggests that the filament may be a dense stellar wind-blown relic of the progenitor star heated by the shock wave.

Figure 10 also shows a high $E_{m}$ belt running through the SNR Puppis A, exactly at the same position as the large-scale filament we analyzed in this paper. Hui \& Becker (2006) suggest that this belt may be due to intervening absorbing material from Vela SNR. Dubner et al. (2013) present a comparison of the X-ray image of SNR Puppis A with the HI column distribution (Reynoso et al. 2003) that shows a stripe with enhanced hydrogen column density $\left(N_{\mathrm{H}} \sim 10^{21} \mathrm{~cm}^{-2}\right)$ in coincidence with the high $E_{m}$ belt, suggesting that dense material might be responsible for absorbing soft X-ray photons. Given the spatial coincidence of the large scale filament with the high absorption feature in Puppis A, it may be possible that the filament, together with the X-ray emitting plasma, also includes cooler and denser material, which may be responsible for such a large absorption. This scenario surely requires further investigation of the lower energy emission from this feature.

In this scenario, the filament can be considered as the projection on the plane of the sky of a nearly spherical shell, likely a wall of a wind-blown bubble. Given the poor spatial resolution of the ROSAT telescope, the width of the shell can be estimated by the XMM-Newton data only (i.e., only in the southern edge of the filament) and is $W \sim 2 \times 10^{17} \mathrm{~cm}$. In the following, we assume that this is the width along the whole filament. We estimate the volume of the X-ray emitting plasma within the photon extraction regions, which we approximated as the volume intercepted by the extraction region on two spherical shells (having width $W$ ) and different radii, $r_{1}=5.0 \times 10^{19} \mathrm{~cm}$ and $r_{2}=4.3 \times 10^{19}$ $\mathrm{cm}$. This can be done by adopting the procedure described in Miceli et al. (2012) (see their Appendix A).

We obtain a density $n_{f i l} \sim 0.182_{-0.015}^{+0.020} \mathrm{~cm}^{-3}$ for the whole filament (region Ext_fil in Fig. 9, analyzed with ROSAT) and $n_{f i l} \sim 0.40 \pm 0.02 \mathrm{~cm}^{-3}$ for the southern edge of the filament (region fil_all in Fig. 5, analyzed with XMM-Newton). This may indicate small (within a factor of $\sim 2$ ) spatial variations in the shell density and/or variations in its width (the assumption of a uniform width may not be strictly valid and this may affect the density estimate). By considering an average density $n_{\text {fil }}=0.2$ $\mathrm{cm}^{-3}$, we also estimated the mass of the spherical shell blown by the wind with external radius $R_{\text {ext }}=4.49 \times 10^{19} \mathrm{~cm}$ and internal radius $R_{\text {int }}=4.47 \times 10^{19} \mathrm{~cm}$, thus finding $M_{\text {shell }}=2.5_{-0.2}^{+0.3} \times 10^{33}$ $\mathrm{g}\left(1.26_{-0.10}^{+0.15} M_{\odot}\right)$.

Considering the projected average distance between the explosion center of Vela SNR and the forward shock in the western region, we obtained a velocity of $v \sim 2 \times 10^{8} \mathrm{~cm} / \mathrm{s}$. Using this velocity and considering an average distance from the explosion center $\left(r_{\text {shell }} \sim 5 \times 10^{19} \mathrm{~cm}\right)$, we found that the shock interacted with the filament structure approximately $8 \mathrm{kyr}$ after the explosion. This value is an upper limit value. Indeed, the filamentary structure was probably dragged by the interaction with the forward shock. It is also possible to get an estimate of the age of this putative wind-blown structure. By using the $r_{\text {shell }}$ value and the characteristic speed of stellar winds in a red supergiant $(\sim 10$ $\mathrm{km} / \mathrm{s}$ ), we determined that the progenitor star started to blow this wind (i.e., entered in its red supergiant phase) 1.6 Myr ago. The onset of the red supergiant phase typically precedes the explosion by $0.5-2 \mathrm{Myr}$; therefore, our time estimate is quite rea- 
sonable and strongly supports the interpretation of the filament as a wind-blown structure produced by the progenitor star.

On the other hand, the high $\mathrm{Ne} / \mathrm{O}$ ratio suggests peculiar abundances for this putative wind-blown shell. A possible explanation for a high $\mathrm{Ne} / \mathrm{O}$ ratio can be $\mathrm{O}$ depletion. In layers where the $\mathrm{CNO}$ cycle is active, the $\mathrm{O}$ abundance strongly decreases (while Ne abundance stays steady). Therefore, large Ne/O abundance ratios are expected in the inner part of the $\mathrm{H}$ layer of a massive star. To blow a wind with enhanced $\mathrm{Ne} / \mathrm{O}$ abundances, it is then necessary for the star to lose its $\mathrm{H}$ envelope, as occurs in Wolf-Rayet stars (WNL-WNE). A Wolf-Rayet star can blow a fast wind that can reach, and impact, the previously ejected material, thus forming a shell with enhanced $\mathrm{Ne} / \mathrm{O}$ abundances, similar to what we observed (Chieffi \& Limongi 2013, Limongi \& Chieffi 2018). In order to test this scenario, it would be necessary to measure the $\mathrm{N}$ and $\mathrm{C}$ abundances in the wind-blown shell. Unfortunately, we cannot obtain significant constraints on these abundances with our data and further investigations are necessary. The detection of a wind-blown relic, in any case, can convey important information on the progenitor star of Vela SNR.

Acknowledgements. The authors are grateful to the referee for very constructive comments and inspiring suggestions. The authors would like to thank A. Chieffi and M. Limongi for the very helpful suggestions regarding the metallicity in stellar winds. MM acknowledges partial support from the Italian Ministry of Research through the "Fondo per il finanziamento delle attività base di ricerca" (FFABR 2017). SO, MM, FB and EG acknowledge financial contribution from the INAF mainstream program. FG and JAC acknowledge support by PIP 0102 (CONICET). JAC is CONICET researcher. This work received financial support from PICT-2017-2865 (ANPCyT). JAC was also supported by grant PID2019105510GB-C32/AEI/10.13039/501100011033 from the Agencia Estatal de Investigación of the Spanish Ministerio de Ciencia, Innovación y Universidades, and by Consejería de Economía, Innovación, Ciencia y Empleo of Junta de Andalucía as research group FQM-322, as well as FEDER funds. FG acknowledges the research programme Athena with project number 184.034.002, which is (partly) financed by the Dutch Research Council (NWO).

\section{References}

Anders, E. \& Grevesse, N. 1989, Geochim. Cosmochim. Acta, 53, 197

Arnaud, K. A. 1996, Astronomical Society of the Pacific Conference Series, Vol. 101, XSPEC: The First Ten Years, ed. G. H. Jacoby \& J. Barnes, 17

Aschenbach, B., Egger, R., \& Trümper, J. 1995, Nature, 373, 587

Bear, E. \& Soker, N. 2018, ApJ, 855, 82

Caraveo, P. A., De Luca, A., Mignani, R. P., \& Bignami, G. F. 2001, ApJ, 561, 930

Chieffi, A. \& Limongi, M. 2013, ApJ, 764, 21

Dodson, R., Legge, D., Reynolds, J. E., \& McCulloch, P. M. 2003, ApJ, 596, 1137

Dubner, G., Loiseau, N., Rodríguez-Pascual, P., et al. 2013, A\&A, 555, A9

García, F., Suárez, A. E., Miceli, M., et al. 2017, A\&A, 604, L5

Greco, E., Miceli, M., Orlando, S., et al. 2018, A\&A, 615, A157

Greco, E., Vink, J., Miceli, M., et al. 2020, A\&A, 638, A101

Hui, C. Y. \& Becker, W. 2006, A\&A, 454, 543

Hwang, U. \& Laming, J. M. 2012, ApJ, 746, 130

Katsuda, S., Mori, K., Petre, R., et al. 2011, PASJ, 63, S827

Katsuda, S. \& Tsunemi, H. 2005, PASJ, 57, 621

Katsuda, S. \& Tsunemi, H. 2006, ApJ, 642, 917

LaMassa, S. M., Slane, P. O., \& de Jager, O. C. 2008, ApJ, 689, L121

Laming, J. M., Hwang, U., Radics, B., Lekli, G., \& Takács, E. 2006, ApJ, 644, 260

Limongi, M. \& Chieffi, A. 2018, ApJS, 237, 13

Lopez, L. A., Pearson, S., Ramirez-Ruiz, E., et al. 2013, ApJ, 777, 145

Lu, F. J. \& Aschenbach, B. 2000, A\&A, 362, 1083

Miceli, M., Bamba, A., Orlando, S., et al. 2017, A\&A, 599, A45

Miceli, M., Bocchino, F., Decourchelle, A., et al. 2012, A\&A, 546, A66

Miceli, M., Bocchino, F., Maggio, A., \& Reale, F. 2005, A\&A, 442, 513

Miceli, M., Bocchino, F., \& Reale, F. 2008a, ApJ, 676, 1064

Miceli, M., Decourchelle, A., Ballet, J., et al. 2008b, Advances in Space Research, 41, 390

Miceli, M., Orlando, S., Reale, F., Bocchino, F., \& Peres, G. 2013, MNRAS, 430, 2864

Orlando, S., Miceli, M., Pumo, M. L., \& Bocchino, F. 2016, ApJ, 822, 22
Orlando, S., Wongwathanarat, A., Janka, H. T., et al. 2021, A\&A, 645, A66 Reynoso, E. M., Green, A. J., Johnston, S., et al. 2003, MNRAS, 345, 671 Slane, P., Lovchinsky, I., Kolb, C., et al. 2018, ApJ, 865, 86

Strüder, L., Briel, U., Dennerl, K., et al. 2001, A\&A, 365, L18

Taylor, J. H., Manchester, R. N., \& Lyne, A. G. 1993, ApJS, 88, 529

Tsebrenko, D. \& Soker, N. 2015, MNRAS, 453, 166

Turner, M. J. L., Abbey, A., Arnaud, M., et al. 2001, A\&A, 365, L27

Tutone, A., Orlando, S., Miceli, M., et al. 2020, A\&A, 642, A67

Vink, J. 2004, New A Rev., 48, 61

Zhou, P. \& Vink, J. 2018, A\&A, 615, A150

Zhou, X., Miceli, M., Bocchino, F., Orlando, S., \& Chen, Y. 2011, MNRAS, 415,

Article number, page 10 of 10 